\title{
Tailor-made directional emission in nanoimprinted plasmonic-based light-emitting devices
}

\author{
G. Lozano, $\dagger^{\text {*a }}$ G. Grzela, ${ }^{a}$ M. A. Verschuuren, ${ }^{b}$ M. Ramezani ${ }^{b}$ and J. Gómez Rivas ${ }^{\text {ac }}$
}

We demonstrate an enhanced and tailor-made directional emission of light-emitting devices using nanoimprinted hexagonal arrays of aluminum nanoparticles. Fourier microscopy reveals that the luminescence of the device is not only determined by the material properties of the organic dye molecules but is also strongly influenced by the coherent scattering resulting from periodically arranged metal nanoparticles. Emitters can couple to lattice-induced hybrid plasmonic-photonic modes sustained by plasmonic arrays. Such modes enhance the spatial coherence of an emitting layer, allowing the efficient beaming of the emission along narrow angular and spectral ranges. We show that tailoring the separation of the nanoparticles in the array yields an accurate angular distribution of the emission. This combination of large-area metal nanostructures fabricated by nanoimprint lithography and light-emitting devices is beneficial for the design and optimization of solid-state lighting systems.

Received 13th March 2014 Accepted 22nd May 2014

DOI: $10.1039 / \mathrm{c} 4 \mathrm{nr} 01391 \mathrm{c}$

www.rsc.org/nanoscale changing the material structure or the chemical composition of very efficient state-of-the-art emitting materials. The field of plasmonics offers a route to control light-matter interactions in the nanoscale with high accuracy through the use of strong local field enhancements near metal nanostructures. ${ }^{21,22}$ It has been found that the emission characteristics of light sources located in the proximity of metal nanoparticles can be strongly modified. ${ }^{23-31}$ While individual metal nanoparticles sustain localized surface plasmon polaritons (LSPPs), an array of such nanoparticles can also support delocalized plasmonic-photonic hybrid states due to the coupling of LSPPs to diffracted or refractive-index guided modes. ${ }^{32}$ Diffractive and waveguide coupling give rise to collective lattice-induced modes (LMs) known as surface lattice resonances (SLRs) ${ }^{33}$ and waveguideplasmon polaritons (WPPs), ${ }^{34}$ respectively. These resonances are responsible for large field enhancements that extend away from the nanoparticles in the volume in which the wavelengthconverting material is distributed..$^{35-41}$ Several experimental studies have addressed the modification of the emission of light sources in the proximity of LMs. These include the directional out coupling of the emission through dipolar and multipolar resonances, ${ }^{\mathbf{4 2 , 4 3}}$ the strong coupling of excitons to LMs, ${ }^{\mathbf{4 4 , 4 5}}$ and lasing. ${ }^{46}$ In turn, a tailor-made control over the angular distribution of the emission of light-emitting devices has not yet been realized.

In this manuscript, we demonstrate that the emission directionality of colour-converting LEDs can be accurately controlled using periodic arrays of metal nanoparticles arranged in a hexagonal lattice. The hexagonal lattice or equilateral triangular lattice is one of the five Bravais bi-dimensional lattices. It shows a six-fold symmetry that facilitates a nearly homogeneous distribution of the emission over the azimuthal
Campus 4, 5656 AE, Eindhoven, The Netherlands

${ }^{b}$ Philips Research, Eindhoven, The Netherlands

${ }^{c}$ COBRA Research Institute, Eindhoven University of Technology, Eindhoven, The Netherlands

$\dagger$ Present address: Instituto de Ciencia de Materiales de Sevilla, Consejo Superior de Investigaciones Científicas-Universidad de Sevilla, Av. Américo Vespucio 49, 41092, Sevilla, Spain. Email: g.lozano@csic.es 
angle. The colour converter consists of a polymer layer doped with a highly efficient dye deposited over an array of $\mathrm{Al}$ nanoparticles. The metal nanostructures are fabricated using a nanoimprint lithography (NIL) technique in combination with a reactive ion etching (RIE) process. NIL is a simple nanofabrication method that has attracted much attention in several fields of research for different applications due to its high resolution and large throughput. ${ }^{4-49}$ A high power blue LED is employed to excite the emitting material. The directionality of the resulting emission of the device is investigated using Fourier microscopy (FM). Excited dye molecules dispersed in the colour-converting layer deposited over the array can couple to LMs, which radiate into the free space. The coherent scattering from periodically arranged metal nanoparticles enables the beaming of most of the emission into small solid angles in defined directions. Here we demonstrate that by tailoring the lattice constant of the array, and consequently the onset of diffraction, it is possible to increase the emission intensity in defined directions and shape the directionality of the colourconverting LEDs using plasmonics. We will show that metal nanostructure arrays act as compact, efficient, diffractive nanooptics to accurately control the angular distribution of light-emitting devices.

\section{Results and discussion}

NIL makes use of nanostructured stamps to mold a resist layer into a three-dimensional shape, thereby transferring a nanostructure from the stamp into the resist. Substrate conformal imprint lithography (SCIL) is a novel NIL technique that uses composite stamps where the nanostructure is in a silicone rubber which is grafted onto a $200 \mu \mathrm{m}$-thin glass sheet. ${ }^{50}$ This allows the faithful replication of nanopatterns over large-area substrates, i.e. up to $20 \mathrm{~cm}$ in diameter. Replicated patterns with details smaller than $10 \mathrm{~nm}$ have been demonstrated using

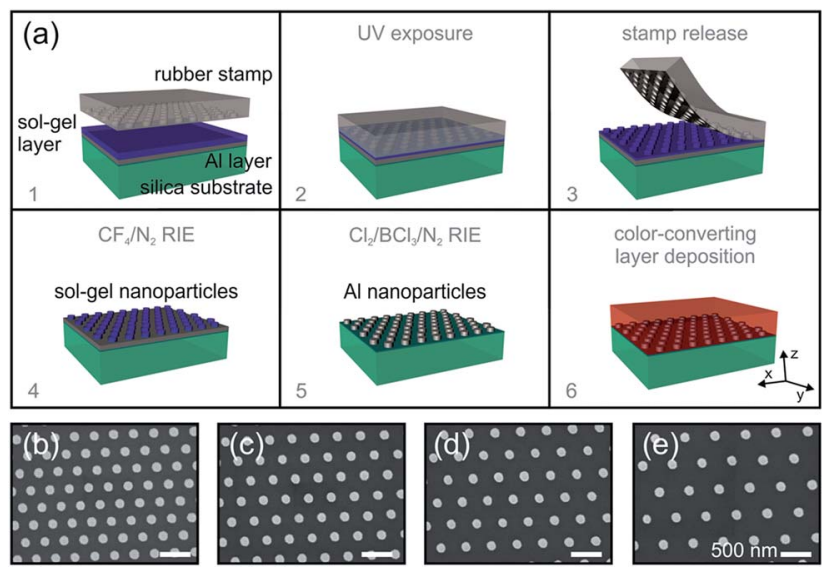

Fig. 1 (a) Schematic of the fabrication process of the plasmonicbased colour-converting layer. (b-e). Scanning electron microscopy images of the top surface of the hexagonal array of Al nanoparticles deposited over the silica substrate. The lattice parameter of the array is (b) $325 \mathrm{~nm}$, (c) $375 \mathrm{~nm}$, (d) $425 \mathrm{~nm}$ and (e) $475 \mathrm{~nm}$. The scale bars represent $500 \mathrm{~nm}$.
NIL. ${ }^{51,52}$ Fig. 1(a) illustrates the fabrication process of a plasmonic-based colour-converting layer, i.e. a dye-doped polymer layer that covers a periodic array of $\mathrm{Al}$ nanoparticles deposited on a glass silica substrate. $\mathrm{Al}$ is chosen as the metal because it exhibits a plasmonic behavior in the blue part of the electromagnetic spectrum and is inexpensive and easy to process. In order to fabricate the arrays of metallic nanoparticles, the substrates are first cleaned with a mixture of $\mathrm{H}_{2} \mathrm{SO}_{4}$ and $\mathrm{H}_{2} \mathrm{O}_{2}$, UV-ozone and then thoroughly rinsed with demineralized water. Then, a $30 \mathrm{~nm}$ layer of $\mathrm{Al}_{2} \mathrm{O}_{3}$, which serves as an etch stop, is deposited by sputtering, after which a $150 \mathrm{~nm}$-thick layer of $\mathrm{Al}$ is deposited using the same method. Before nanoimprinting, the Al samples are cleaned with oxygen plasma. Then, a ca. $60 \mathrm{~nm}$-thick sol-gel resist is applied by spin-coating. Shortly after the spin-coating cycle is finished, the SCIL stamp is applied into the resist. The stamp consists of several hexagonal arrays of holes in which the interparticle distance ranges from $300 \mathrm{~nm}$ to $475 \mathrm{~nm}$ in steps of $25 \mathrm{~nm}$. The sol-gel was cured by applying $365 \mathrm{~nm}$ UV light on the stamp. Next the stamp is carefully released and the sample is given a post bake at $150{ }^{\circ} \mathrm{C}$. This results in the production of silica sol-gel nanoparticles of $80 \mathrm{~nm}$ to $150 \mathrm{~nm}$ in diameter and $c a .100 \mathrm{~nm}$ in height on the top of the Al layer. The nanoimprinted sol-gel patterns are subsequently transferred into the Al layer by a RIE technique. First the residual layer beneath the sol-gel particles is removed using $\mathrm{CF}_{4} / \mathrm{N}_{2}$. Approximately $55 \mathrm{~nm}$ of sol-gel is removed, which ensures that the areas between the sol-gel nanoparticles are clear of resist and the Al layer is exposed. The sample is then transferred under vacuum to a second RIE chamber, which uses $\mathrm{Cl}_{2}$ and $\mathrm{BCl}_{3}$ to etch, and $\mathrm{N}_{2}$ and $\mathrm{CH}_{4}$ to passivate. Fig. 1(b)-(e) show scanning electron microscopy pictures of the top view of the nanoimprinted $\mathrm{Al}$ arrays, which are characterized by lattice constants of $a=325 \mathrm{~nm}, a=375 \mathrm{~nm}, a=425 \mathrm{~nm}$ and $a=475$ $\mathrm{nm}$, respectively. Every aluminium nanoparticle shows a very thin layer of $\mathrm{Al}_{2} \mathrm{O}_{3}$ that prevents the metallic nanoparticle from further oxidation. The combination of NIL and RIE allows a high-throughput patterning of large-area nanoplasmonic structures with great precision and at low costs. Finally, over the Al nanoparticle arrays, a $700 \mathrm{~nm}$-thick polystyrene layer doped with dye molecules (BASF, Lumogen F Red 305) at 3\% of mass fraction, which acts as a colour-converting layer, is deposited by spin coating. This dye is stable, bright in the red part of the spectrum where the human eye is most sensitive, and shows a close-to-one photoluminescence quantum yield, all of which make it highly suitable for SSL applications. The thickness of the layer and the concentration of the dye molecules in the polymer matrix determine the absolute fraction of blue LED light converted into red, being around $25 \%$ in the system herein investigated.

The experimental extinction spectrum (defined as $1-T_{0}$, being $T_{0}$ the zeroth order transmission) at normal incidence of the hexagonal array with a lattice constant of $475 \mathrm{~nm}$ is shown in Fig. 2(a) to illustrate the plasmonic resonances of such arrays. The two narrow peaks observed at $\lambda=616 \mathrm{~nm}\left(\lambda_{1}\right)$ and $\lambda=$ $631 \mathrm{~nm}\left(\lambda_{2}\right)$ are associated with LMs supported by the structure. The photoluminescence spectra of the colour-converting layer deposited over the array (solid black curve) and on the top of the 
(a)

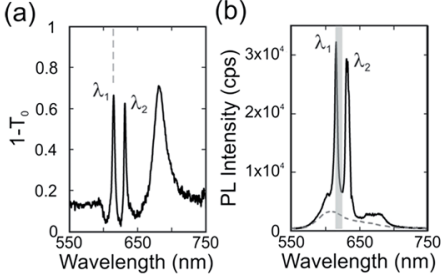

(c)

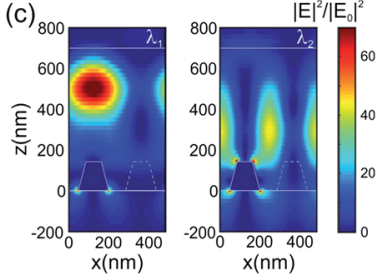

Fig. 2 (a) Extinction spectrum measured at normal incidence from a hexagonal array with a lattice constant of $475 \mathrm{~nm}$. The gray dashed line indicates the onset of the first diffraction order calculated in a medium with a refractive index of 1.5 . This value is obtained as a fitting to the extinction peak observed at $\lambda=616 \mathrm{~nm}$. (b) Emission spectrum measured at normal incidence from a colour-converting (organic dyedoped polymer) layer deposited over the array (solid black curve) and over a flat glass substrate (dashed gray curve). $445 \mathrm{~nm}$ light is employed to excite the colour-converting material. (c) Spatial distribution of the electric field intensity calculated using FDTD in a unit cell of the array. Simulations consider a plane wave with a wavelength of $\lambda_{1}$ (left) and $\lambda_{2}$ (right) that illuminates the hexagonal array at normal incidence. The metallic nanoparticles and the interfaces that separate the different dielectric media are highlighted using gray lines.

glass substrate (dashed gray curve) are displayed in Fig. 2(b). The presence of the hexagonal array results in: (i) a strong modification of the shape of the emission spectrum of the dye molecules and (ii) an enhancement of the emission intensity. Specifically, the photoluminescence intensity increases significantly over narrow spectral regions as a result of the coupling of the emission to these LMs, which spectrally overlap with the emission spectrum of the colour-converter. The distinct spatial distribution of the total electric field intensity enhancement confirms the different nature of each LM. Fig. 2(c) shows the numerical finite-difference time domain (FDTD) simulations: of the spatial distribution of the electric field intensities at $\lambda_{1}$ and $\lambda_{2}$, corresponding to a WPP and a SLR, respectively. In contrast to the WPP, the field associated with the SLR originates from diffractive coupling in the plane of the array, decaying the field intensity evanescently out of that plane. The hybridized nature of the LMs is clearly observed. Although the field is enhanced in the proximity of the nanoparticles, as expected for a LSPP, it extends over the polymer layer (in which the dye molecules are distributed). This leads to a significant modification of the emission over narrow spectral ranges in the normal direction.

\footnotetext{
\$ FDTD Solutions (Lumerical Solutions Inc.) http://www.lumerical.com/. In order to calculate the transmittance of the periodic arrays, the simulation volume was equal to $a \times a \times 1 \mu \mathrm{m}$ and periodic boundary conditions were used in the in-plane directions and perfectly matched layer conditions in the $z$ direction. The light source was a broadband (450-750 nm) plane wave, with normal incidence to the substrate from the top. The mesh grid was set to $5 \mathrm{~nm}$ over the entire simulation volume, with a refinement of $1 \mathrm{~nm}$ over the volume occupied by the nanoparticle. The transmittance spectra were directly simulated with a frequency-domain transmission monitor. To calculate the spatial distribution of the local electric field intensity, a three-dimensional frequency-domain field monitor and a three-dimensional refractive index monitor that contain both the layer of refractive index 1.59 and the metallic nanoparticle were employed. The optical constants of $\mathrm{Al}$ were taken from ref. 57.
}

In order to investigate the angular emission of the lightemitting device, FM is employed. Images of the intensity distribution on the back focal plane (BFP, the so-called Fourier plane) of a high numerical aperture (NA) objective are recorded on a charge-coupled device (CCD) camera. ${ }^{53}$ Fourier images contain information about the intensity of light emitted in different directions from the normal to the substrate. Fig. 3 shows a sketch of the light-emitting device and a ray tracing diagram for three different emission angles: $\theta_{1}, \theta_{2}$ and $\theta_{3}$, to illustrate the principle of FM. Every plane wave emitted from the object plane reaches the same point at the BFP. In particular, light emitted at an angle $\theta_{1}\left(\theta_{2}\right)$ is collected at a distance $x_{1}\left(x_{2}\right)$ from the center of the Fourier image, wherein light emitted in the normal direction from the sample is detected. The NA of the objective determines the largest angle that can be picked up by the objective according to $\theta_{\max }=\arcsin (\mathrm{NA})$. Thus, light emitted with an angle $\theta_{3}>\theta_{\max }$ will not reach the BFP nor the detector. FM enables a fast and accurate quantitative characterization of the directionality of the emission of SSL devices.

An electrically driven high-power blue LED $\left(\lambda_{\mathrm{ex}}=445 \pm 15\right.$ $\mathrm{nm}$ ) is employed to optically pump the colour-converter now featuring a nanoparticle array. The emission originated in the colour-converting layer is collected in the Fourier plane. No polarization selection is carried out, since polarization dependency is generally unwanted in SSL systems. The Fourier images are plotted in polar coordinates, where the radius represents the elevation angle of emission $(\theta)$ and the polar angle corresponds to the azimuthal angle of emission $(\phi)$ - see the inset in Fig. 4. The large NA of the microscope objective (0.95) allows collection of light at large angles of emission limited by $\theta_{\max }=72 \mathrm{deg}$. from the normal to the sample.

Fig. 4(a) displays the Fourier image of the unpolarized emission (in units of counts per $0.1 \mathrm{~s}$ ) from the colour-converting layer deposited over the flat dielectric substrate that will be considered as the reference colour-converting LED. A $10 \mathrm{~nm}$ band-pass filter is employed to spectrally select the emission at

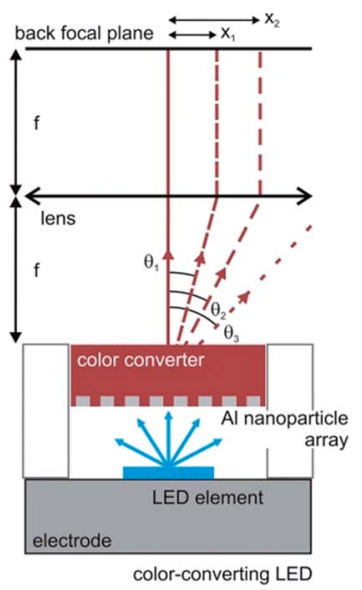

Fig. 3 Schematic of the colour-converting light emitting diode (LED). The upper part of the panel illustrates the working principle of Fourier microscopy. Light emitted by a colour-converting LED at an angle $\theta_{1}$ $\left(\theta_{2}\right)$ is collected at the position $x_{1}\left(x_{2}\right)$ in the back focal plane of the lens. Light emitted at an angle $\theta_{3}$ is not picked up by the lens. 

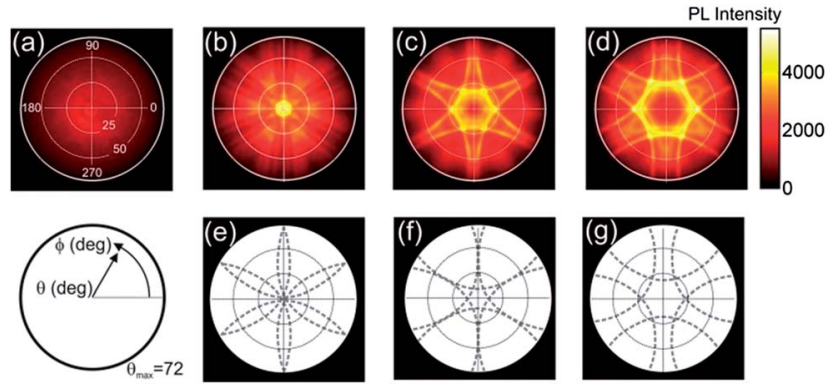

Fig. 4 (a) Fourier image of the unpolarized emission from the nonplasmonic colour converting layer, i.e. a $700 \mathrm{~nm}$-thick dye-doped polymer layer deposited over a flat silica substrate. (b, c and d) Fourier images of the unpolarized emission of the same colour-converting layer deposited over a hexagonal array of Al nanoparticles with lattice constants (b) $475 \mathrm{~nm}$, (c) $425 \mathrm{~nm}$ and (d) $375 \mathrm{~nm}$. They are taken using a band pass filter with a central wavelength of $620 \mathrm{~nm}$ and a bandwidth of $10 \mathrm{~nm}$ at full width at half maximum. An electrically driven blue LED $\left(\lambda_{\text {ex }}=445 \pm 15 \mathrm{~nm}\right)$ is employed to optically excite the colour-converting layer. The outermost circle indicates the maximum angle $\left(\theta_{\max }\right.$ $=72$ deg.) that can be collected using an objective with a numerical aperture of 0.95. (e, $f$ and $g$ ) Rayleigh anomalies (gray dashed curves) calculated for the $( \pm 1,0),(0, \pm 1),(-1,1)$ and $(1,-1)$ beams diffracted by a hexagonal array with lattice constants (e) $a=475 \mathrm{~nm}$, (f) $a=425 \mathrm{~nm}$ and $(\mathrm{g}) \mathrm{a}=375 \mathrm{~nm}$, in a medium with a refractive index of 1.5 .

$\lambda=620 \mathrm{~nm}$, a relevant spectral range to achieve warm white light. At $640 \mathrm{~nm}$, the eye sensitivity curve drops below 15\% of its maximum located at $555 \mathrm{~nm}$, being the red light emitted above $640 \mathrm{~nm}$ rather useless for general illumination purposes. The emission pattern shows no particular directional features. This is expected for a random distribution of dye molecules deposited over a flat surface, which behaves as a Lambertian source. The emission of such a layer deposited over hexagonal arrays with lattice constants $a=475 \mathrm{~nm}, a=425 \mathrm{~nm}$ and $a=375 \mathrm{~nm}$ is shown in Fig. 4(b), (c) and (d), respectively. The emission is collected in the same spectral range used for the reference measurement $(\lambda=620 \pm 5 \mathrm{~nm})$. The effect of the combination of periodic arrays of metal nanoparticles with a colour-converting layer is twofold: (i) an enhancement of the amount of light extracted from the emitting layer and (ii) a drastic modification of the angular distribution of the emission. Fourier images reveal a strong directional response of the emission with bands of bright luminescence that are associated with latticeinduced modes. These features depend on the separation between metal nanoparticles in the array. Clear six fold symmetry is observed due to the geometry of the hexagonal lattice. Note that the bands of high emission intensity are superimposed to a background that originates from the emission of the dye molecules that do not couple to any mode supported by the array. The higher symmetry of the hexagonal lattice, compared to the most widely employed square lattice, is beneficial for SSL since it provides a more even distribution of the light emission over the azimuthal angle. In order to gain more physical insight into the different emission patterns attained for the arrays with different interparticle distances, we calculate the onset of diffraction or the Rayleigh anomalies (RAs) for the different diffracted orders supported by the investigated lattices. Fig. 4(e), (f) and (g) show the RAs calculated for the $( \pm 1,0),(0, \pm 1),(-1,1)$ and $(1,-1)$ orders diffracted by a hexagonal array with lattice constants $a=475 \mathrm{~nm}, a=$ $425 \mathrm{~nm}$ and $a=375 \mathrm{~nm}$, respectively, at $\lambda=620 \mathrm{~nm}$ in a medium with a refractive index of 1.5 . The coupling of the light emitted by the dye molecules distributed in the colour-converting layer to LMs supported by the array gives rise to narrow bands of enhanced directional emission that follow the dispersion of the RAs. We note that the experimental emission consists of double nearly parallel bands - clearly seen in Fig. 4(d). These double bands are associated with SLRs and WPPs and have been reported in ref. 29. They are attributed to the excitation of LMs induced, respectively, by in-plane scattering of diffractive orders and waveguide modes supported in the dye layer.

A periodic array of metal nanoparticles behaves as a phased array of optical antennas in which the radiation pattern of subwavelength sources is modified by the coherent scattering of the periodically spaced metallic nanoparticles. Light coupled out by the array interferes constructively in defined directions, resulting in an efficient beaming of the light emitted by the colourconverting layer. The beaming of the emission can be also interpreted in terms of an increased spatial coherence of point sources due to their coupling to the spatially extended LMs. ${ }^{54}$ To assess the directionality of the emission by the arrays, the emitted intensity as observed from the Fourier images shown in Fig. 4(a)-(d) is integrated over $\phi$. Fig. 5(a) displays the polar plots of the azimuthally integrated emitted intensity in which each curve is normalized to its maximum. It is observed that tailoring the interparticle distance in the periodic array permits the accurate control of the beaming angle, which is defined as the direction at which most of the red light intensity is beamed. Specifically, the beaming angle increases as the lattice constant of the array decreases. The emission spectrum of the colourconverting layer overlaps with the LM at a larger angle when the separation between particles in the array diminishes. For comparison, the gray filled area in Fig. 5(a) is a polar plot of the azimuthally integrated Fourier image presented in Fig. 4(a), which displays the emission of the colour-converting layer deposited over a flat glass substrate. A careful inspection of this curve shows a small modulation of the intensity, which is an artefact that originates from the imaging system in the experimental setup. Fig. 5(b) displays the beaming angle measured as a function of the lattice parameter for different arrays (black squares). The RA of the $(1,0)$ diffracted beam calculated at $\lambda=$ $620 \mathrm{~nm}$ as a function of the lattice constant and the elevation angle for $\phi=0 \mathrm{deg}$. is also shown as a dashed curve in the same plot as a guide to the reader. The shift of the measurements with respect to the RA is the result of the coupling between the plasmonic and the photonic mode. Such coupling gives rise to the LMs to which the emission of the dye couples. ${ }^{55}$ This analysis reveals that an accurate control of the periodicity of the plasmonic array allows tailoring the angular distribution of the emission of light-emitting devices.

To further elucidate the modification of the spontaneous emission intensity, in Fig. 5(c) we plot the photoluminescence directional enhancement (PLDE), i.e., the emission intensity of the plasmonic colour converter at the beaming angle 
(a)

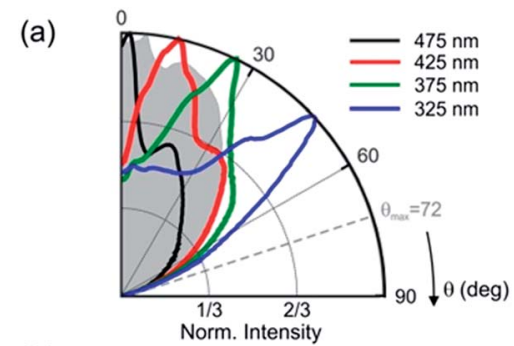

(b)

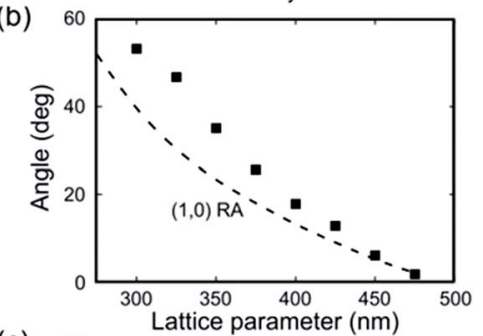

(c)

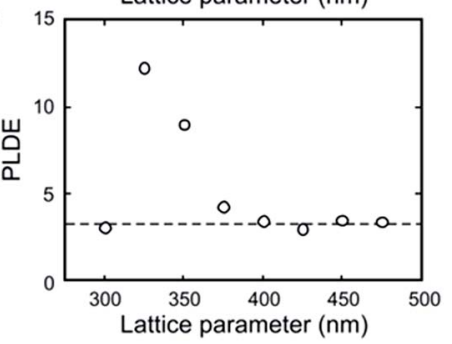

Fig. 5 (a) Angular distribution of the unpolarized emission (coloured solid curves) in the wavelength range $\lambda=620 \pm 5$ from a $700 \mathrm{~nm}$ thick dye-doped polymer, acting as a colour-converting layer, deposited over a hexagonal array of Al nanoparticles with lattice constants 475 $\mathrm{nm}$ (black curve), $425 \mathrm{~nm}$ (red curve), $375 \mathrm{~nm}$ (green curve) and 325 $\mathrm{nm}$ (blue curve). The grey filled area is the angular distribution of the unpolarized emission of the colour-converting layer in the absence of the metallic nanoparticles. An electrically driven blue LED emitting at $\lambda_{\text {ex }}=445 \pm 15 \mathrm{~nm}$ is employed to optically excite the colour-converting layer. Each radiation pattern is normalized to its maximum intensity. The dashed gray line corresponds to the maximum angle $\left(\theta_{\max }=72\right.$ deg. $)$ that can be collected in our setup. (b) Emission angle at which most of the intensity is beamed as a function of the lattice constant of the hexagonal array (black squares). The angular position of the Rayleigh anomaly calculated in a medium with a refractive index of 1.5 for the $(1,0)$ order diffracted by a hexagonal array as a function of the lattice constant (dashed curve). (c) Photoluminescence directional enhancement (PLDE) defined as the emission from the plasmonic colour-converting layer at $\lambda=620 \pm 5 \mathrm{~nm}$ normalized to the emission of the colour-converting layer on the top of the flat silica substrate as a function of the lattice constant of the hexagonal array. The horizontal dashed line is a guide to the eye.

normalized by the emission of the colour converter at the same angle in the absence of metallic nanoparticles, as a function of the lattice parameter of the hexagonal array. In general, the PLDE can be factorized into two contributions assuming that the excitation and the emission are two independent processes occurring at different wavelengths ( $\lambda_{\mathrm{ex}}$ and $\lambda$, respectively). ${ }^{56,42}$ Thus, the PLDE can be expressed as

$$
\operatorname{PLDE}\left(\lambda_{\mathrm{ex}}, \Omega_{\mathrm{ex}}, \lambda, \Omega\right)=\frac{\int_{V} \eta(\vec{r}, \lambda, \Omega)\left|E\left(\vec{r}, \lambda_{\mathrm{ex}}, \Omega_{\mathrm{ex}}\right)\right|^{2} \mathrm{~d} V}{\int_{V} \eta_{\mathrm{ref}}(\vec{r}, \lambda, \Omega)\left|E_{\mathrm{ref}}\left(\vec{r}, \lambda_{\mathrm{ex}}, \Omega_{\mathrm{ex}}\right)\right|^{2} \mathrm{~d} V}
$$

where $V$ is the volume of the colour-converting layer. $\Omega_{\mathrm{ex}}$ is the solid angle associated with the elevation and the azimuthal angle of excitation, $\left|E\left(\vec{r}, \lambda_{\mathrm{ex}}, \Omega_{\mathrm{ex}}\right)\right|^{2}$ is the local field intensity at the wavelength $\lambda_{\text {ex }}$ and at the position $\vec{r}$ where each dye molecule is located. $\left|E_{\text {ref }}\left(\vec{r}, \lambda_{\text {ex }}, \Omega_{\text {ex }}\right)\right|^{2}$ corresponds to the local field intensity in the absence of the hexagonal array. The term $\eta(\vec{r}, \lambda$, $\Omega$ ) includes the effect of the modified local density of optical states at $\lambda$ to which a dye molecule can decay due to the presence of the hexagonal array and the out coupling of this emission to free space radiation in the solid angle $\Omega . \eta_{\text {ref }}(\vec{r}, \lambda, \Omega)$ represents the same physical quantity as $\eta$, but in the absence of the array. The geometry of the device is such that the colourconverting layer is mainly illuminated along the normal direction to the array. We have performed 3D FDTD numerical simulations (not shown here) in order to elucidate the influence of the excitation enhancement contribution, given by $\int_{V}\left|E\left(\vec{r}, \lambda_{\mathrm{ex}}, \Omega_{\mathrm{ex}}\right)\right|^{2} \mathrm{~d} V$ $\int_{V}\left|E_{\text {ref }}\left(\vec{r}, \lambda_{\text {ex }}, \Omega_{\text {ex }}\right)\right|^{2} \mathrm{~d} V$. The calculations reveal that this contribution is approximately 4 -fold when the lattice parameter is $325 \mathrm{~nm}$. For this interparticle distance, the blue LED light couples very efficiently to LMs supported by the array at $\lambda_{\text {ex }}$, leading to the largest PLDE (12-fold). For the other lattices, in which the effect of the excitation enhancement is negligible, the PLDE is $\sim 3.5$-fold. We attribute most of this overall enhancement to an improved out coupling of the emission from the colour-converting layer. The metallic nanoparticle array acts as an efficient scattering layer that facilitates light extraction from the dye-doped polymer layer, preventing light guiding in the substrate.

\section{Conclusions}

In conclusion, using Fourier microscopy we have demonstrated that large-area nanoimprinted hexagonal plasmonic lattices strongly shape the directionality of the emission of light-emitting devices. By controlling the separation between metal nanoparticles in the array, we have proven an enhanced directional emission from a random distribution of dye molecules in a narrow spectral range. These molecules emit with a close-toone photoluminescence quantum yield in the red part of the visible spectrum where the human eye is most sensitive. We envisage that the use of nanoimprinted metallic arrays will provide a wealth of new opportunities for the design and optimization of solid-state lighting devices in which the control over directionality and colour plays a major role.

\section{Acknowledgements}

We are thankful to Said R. K. Rodríguez, Tommy Barten and Manuela Lunz for stimulated discussions. This work is part of the research program of the Foundation for Fundamental Research on Matter (FOM), which is financially supported by the Netherlands Organization for Fundamental Research (NWO). It is also part of an industrial partnership program between Philips and FOM. It is also supported in part by 
NanoNextNL, a micro and nanotechnology consortium of the Government of the Netherlands and 130 partners.

\section{Notes and references}

1 B. O'Regan and M. Gratzel, Nature, 1991, 353, 737.

2 E. F. Schubert and J. K. Kim, Science, 2005, 308, 1274.

3 A. S. Aricò, P. Bruce, B. Scrosati, J.-M. Tarascon and W. van Schalkwijk, Nat. Mater., 2005, 4, 366.

4 A. Zukauskas, M. S. Shur and R. Caska, Introduction to Solidstate Lighting, John Wiley \& Sons, 2002.

5 D. A. Steigerwald, J. C. Bhat, D. Collins, R. M. Fletcher, M. O. Holcomb, M. J. Ludowise, P. S. Martin and S. L. Rudaz, IEEE J. Sel. Top. Quantum Electron., 2002, 8, 310. 6 J. Y. Tsao, Solid-state lighting: lamps, chips, and materials for tomorrow, IEEE Circ. Dev. Mag., 2004, 20, 28.

7 B. W. D'Andrade and S. R. Forrest, Adv. Mater., 2004, 16, 585.

8 J. M. Phillips, M. E. Coltrin, M. H. Crawford, A. J. Fischer, M. R. Krames, R. M. Mach, G. O. Mueller, Y. Ohno, L. E. S. Rohwer, J. A. Simmons and J. Y. Tsao, Laser Photonics Rev., 2007, 1, 307.

9 P. Schlotter, R. Schmidt and J. Schneider, Appl. Phys. A: Mater. Sci. Process., 1997, 64, 417.

10 N. C. Hu, C. C. Wu, S. F. Chen and H. C. Hsiao, Appl. Opt., 2008, 47, 3423.

11 R. Mueller-Mach, G. O. Mueller, M. R. Krames, O. B. Shchekin, P. J. Schmidt, H. Bechtel, C.-H. Chen and O. Steigelmann, Phys. Status Solidi RRL, 2009, 3, 215.

12 S. Pimputkar, J. S. Speck, S. P. Den Baars and S. Nakamura, Nat. Photon., 2009, 3, 180.

13 N. C. George, K. A. Denault and R. Seshadri, Annu. Rev. Mater. Res., 2013, 43, 481.

14 Z. Zhenrong, H. Xiang and L. Xu, Appl. Optics, 2009, 48, 6627.

15 S. Wang, K. Wang, F. Chen and S. Liu, Opt. Express, 2011, 19, A716.

16 I. Moreno, Opt. Lett., 2012, 37, 839.

17 S. L. Hsiao, N. C. Hu and H. Cornelissen, Opt. Express, 2013, 21, A250.

18 C. C. Hsieh, Y. H. Li and C. C. Hung, Appl. Optics, 2013, 52, 5221.

19 J. J. Wierer, A. David and M. M. Megens, Nat. Photon., 2009, $3,163$.

20 G. Lozano, D. J. Louwers, S. R. K. Rodriguez, S. Murai, O. T. A. Jansen, M. A. Verschuuren and J. Gómez Rivas, Light: Sci. Appl., 2013, 2, e66.

21 A. Polman, Science, 2008, 7, 868.

22 P. Biagioni, J. S. Huang and B. Hecht, Rep. Prog. Phys., 2012, 75, 024402.

23 K. Okamoto, I. Niki, A. Shvartser, Y. Narukawa, T. Mukai and A. Scherer, Nat. Mater., 2004, 3, 601.

24 P. Anger, P. Bharadwaj and L. Novotny, Phys. Rev. Lett., 2006, 96, 113002.

25 S. Kühn, U. Hakanson, L. Rogobet and V. Sandoghdar, Phys. Rev. Lett., 2006, 97, 017402.

26 O. L. Muskens, V. Giannini, J. A. Sánchez-Gil and J. Gómez Rivas, Nano Lett., 2007, 7, 2871.
27 H. Iwase, D. Englund and J. Vučković, Opt. Express, 2008, 16, 426.

28 A. Kinkhabwala, Z. Yu, S. Fan, Y. Avlasevich, K. Mullen and W. E. Moerner, Nat. Photon., 2009, 3, 654.

29 A. G. Curto, G. Volpe, T. H. Taminiau, M. P. Kreuzer, R. Quidant and N. F. van Hulst, Science, 2010, 329, 930.

30 H. Aouani, O. Mahboub, E. Devaux, H. Rigneault, T. W. Ebbesen and J. Wenger, Nano Lett., 2011, 11, 2400.

31 L. Langguth, D. Punj, J. Wenger and A. F. Koenderink, ACS Nano, 2013, 7, 8840.

32 S. Murai, M. A. Verschuuren, G. Lozano, G. Pirruccio, S. R. K. Rodriguez and J. Gómez Rivas, Opt. Express, 2013, 21, 4250.

33 F. J. García de Abajo and J. J. Sáenz, Phys. Rev. Lett., 2005, 95, 233901.

34 A. Christ, S. G. Tikhodeev, N. A. Gippius, J. Kuhl and H. Giessen, Phys. Rev. Lett., 2003, 91, 183901.

35 F. J. García de Abajo, Rev. Mod. Phys., 2007, 79, 1267.

36 K. T. Carron, W. Fluhr, M. W. Meier, A. Wokaun and H. W. Lehman, J. Opt. Soc. Am. B, 1986, 3, 430.

37 B. Auguie and W. L. Barnes, Phys. Rev. Lett., 2008, 101, 143902.

38 V. G. Kravets, F. Schedin and A. N. Grigorenko, Phys. Rev. Lett., 2008, 101, 087403.

39 Y. Chu, E. Schonbrun, T. Yang and K. B. Crozier, Appl. Phys. Lett., 2008, 93, 181108.

40 G. Vecchi, V. Giannini and J. Gómez Rivas, Phys. Rev. B: Condens. Matter Mater. Phys., 2009, 80, 201401.

41 W. Zhou and T. W. Odom, Nat. Nanotechnol., 2011, 6, 423.

42 G. Vecchi, V. Giannini and J. Gómez Rivas, Phys. Rev. Lett., 2009, 102, 146807.

43 V. Giannini, G. Vecchi and J. Gómez Rivas, Phys. Rev. Lett., 2010, 105, 266801.

44 S. R. K. Rodríguez and J. Gómez Rivas, Opt. Express, 2013, 21, 27411.

45 A. I. Vakeväinen, R. J. Moerland, H. T. Rekola, A.-P. Eskelinen, J.-P. Martikainen, D.-H. Kim and P. Törma, Nano Lett., 2014, 14, 1721.

46 W. Zhou, M. Dridi, J. Yong Suh, C. H. Kim, D. T. Co, M. R. Wasielewski, G. C. Schatz and T. W. Odom, Nat. Nanotechnol., 2013, 8, 506.

47 S. Y. Chou, P. R. Krauss and P. J. Renstrom, Appl. Phys. Lett., 1996, 67, 3114.

48 L. J. Guo, Adv. Mater., 2007, 19, 495.

49 S. H. Ahn and L. J. Guo, ACS Nano, 2009, 3, 2304.

50 R. Jia, M. Hornung, M. A. Verschuuren, R. van de Laar, J. van Eekelen, U. Plachetk, M. Moeller and C. Moormann, Microelectron. Eng., 2010, 87, 963.

51 F. Hua, Y. Sun, A. Gaur, M. A. Meitl, L. Bilhaut, L. Rotkina, J. Wang, P. Geil, M. Shim, J. A. Rogers and A. Shim, Nano Lett., 2004, 4, 2467.

52 C. Peroz, S. Dhuey, M. Cornet, M. Vogler, D. Olynick and S. Cabrin, Nanotechnology, 2012, 23, 015305.

53 G. Grzela, R. Paniagua-Domínguez, T. Barten, Y. Fontana, J. A. Sánchez-Gil and J. Gómez Rivas, Nano Lett., 2012, 12, 5481. 
54 L. Shi, T. K. Hakala, H. T. Rekola, J.-P. Martikainen, R. 56 M. Ringler, A. Schwemer, M. Wunderlich, A. Nichtl, J. Moerland and P. Törmä, Phys. Rev. Lett., 2014, 112, K. Kurzinger, T. A. Klar and J. Feldmann, Phys. Rev. Lett., 153002. 2008, 100, 203002.

55 S. Zou, N. Janel and G. C. Schatz, J. Chem. Phys., 2004, 120, 57 E. D. Palik, Handbook of Optical Constants of Solids, 10871. Academic, New York, 1985. 